This is a postprint version of the following published document:

Jerez, B., et al. Gain-switching injection-locked dual optical frequency combs: characterization and optimization, In: Optics letters, 41(18), Sept. 2016, Pp. 4293-4296

DOI: https://doi.org/10.1364/OL.41.004293

(C) 2016 Optical Society of America 


\title{
Gain-switching injection-locked dual optical frequency combs: characterization and optimization
}

\author{
Borja Jerez, ${ }^{*}$ Pedro Martín-Mateos, Estefanía Prior, Cristina de Dios, and Pablo Acedo \\ Electronics Technology Department, Universidad Carlos III de Madrid, Leganés 28911, Spain \\ ${ }^{*}$ Corresponding author: bjerez@ing.uc3m.es
}

In this work, the generation of dual optical frequency combs based on gain-switching and optical injection locking is experimentally examined. The study reveals that an effective process of optical injection can lead to optimized RF combs in terms of span and signal-to-noise ratio. The system also minimizes the overlap of lines and reduces the number of optical components involved, eliminating the need for any external modulator (electro-optic, acousto-optic). The validation of the system was performed as a dual-comb spectrometer, which allowed for determination of the absorption and dispersion profiles of the molecular transition of $\mathbf{H}^{\mathbf{1 3}} \mathbf{C N}$ at $\mathbf{1 5 3 8 . 5 2 3} \mathbf{n m}$. () 2016 Optical Society of America

The process of gain-switching (GS) [1,2] consists of the sudden switch of the laser gain above and below the laser threshold in order to induce a pulsed optical output (and hence, an optical frequency comb, OFC). This has proven to be an interesting approach to OFC generation, due to its compactness, ease and precise control of frequency spacing, high correlation between optical lines, and cost-efficiency. Concurrently, optical injection locking (OIL) is also a largely-used, well-established technique whose prime attribute relies on the conferral of phase locking between two unconnected laser sources [3]. In line with the classic OIL scheme, a slave (injected) laser can inherit the linewidth and optical properties of a master laser [4] and also track any phase variation which takes place into the master [5].

Both methods (GS and OIL) have also proven to be advantageous when implemented in conjunction. With the configuration of a gain-switched slave laser, improvements in terms of signal mode suppression ratio [6], transfer of low linewidth [7], reduction of temporal jitter [8], generation of OFCs with different noise properties [9], and broadening in the locking range [10] have been successfully reported. The research on this topic has also demonstrated the importance of the diode laser technology. Diverse comparisons carried out between
Fabry-Perot (FP) [7,11], discrete mode and distributed feedback lasers (DFBs) [8,12], and, more recently, VCSELs [13] illustrate the importance of the role played by the laser source. Additionally, some recent work has also validated the feasibility of monolithically integrated injection-locked gain-switched OFCs [14-16].

Very recently, the fundamental principles of GS-OIL techniques have been further deployed in dual optical frequency comb architectures [17]. Dual-OFC schemes are based on the down conversion of a pair of OFCs with a narrow difference in their repetition frequencies, $f_{\mathrm{REP}}$. When both combs impinge on a photodetector, the result in the RF domain is a pulse train arising from the interference of the optical pulses, which ultimately leads to an emanating comb in the RF domain, which contains the spectral information previously encoded in the optical spectra. The eminence of this detection arrangement for OFCs relies on its inherent optical capabilities, such as spectral resolution, wide broadband, real-time measurements, and high signal-to-noise ratio (SNR), which have made them extensively employed in spectroscopy [18-25] and, more recently, increasingly utilized in other applications ranging from analysis of optical components $[22,26]$ or fiber Bragg grating sensors [27] to the characterization of arbitrary waveforms [28], among others.

In this Letter, we present an experimental study of the implementation of dual-OFCs (dual-combs) based on the principles of GS-OIL. The outcome of the analysis is two-fold: first, it is possible to take full advantage of the asymmetric character of the gain-switched OFCs and optical injection process to optimize the way the information is recovered at the RF domain (RF comb) without the necessity of an additional frequency shift in one of the OFCs to avoid mode-averaging, as reported previously in continuous-wave (CW) based dual-comb schemes [21-25]. Second, it is doable to maximize the number of teeth and their SNR in the portion of the asymmetric spectrum of interest with careful optimization of the optical injection mechanism. Therefore, most of the lines of the gain-switched OFCs are directly recovered in the RF domain (instead of only half of the lines due to averaging). This way, we demonstrate a dual-comb architecture which preserves the capabilities of previously validated dual-comb schemes but with a reduction in 
(a)

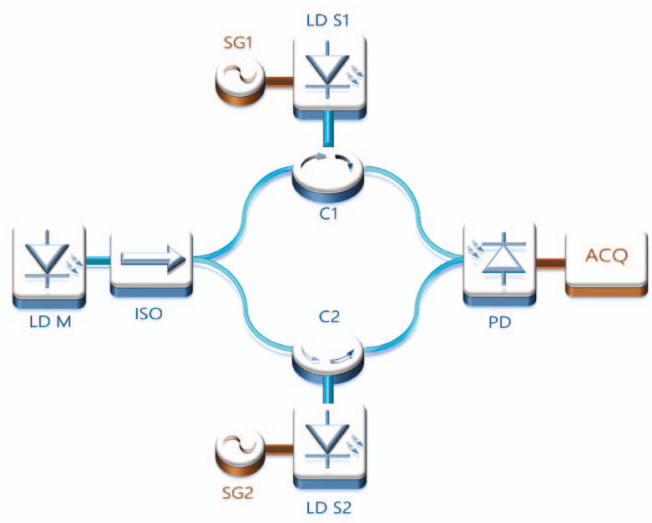

(b)

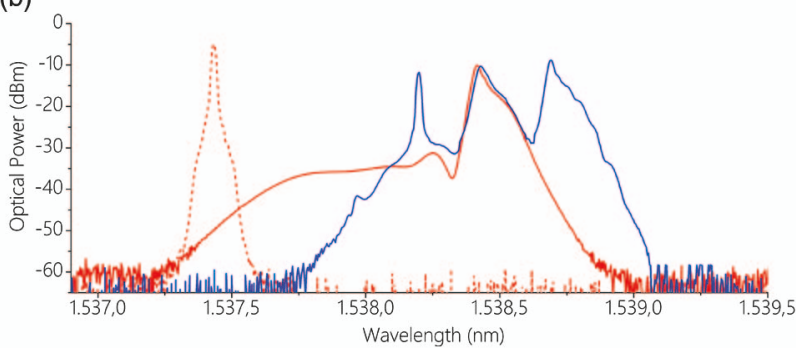

Fig. 1. GS-OIL based dual-comb. (a) Experimental (reduced) setup: LD M: Laser diode-master; ISO, optical isolator; C1/2, circulators; LD S1/2, laser diode slaves; SG1/2, signal generators; PD, photodetector; ACQ, acquisition system. (b) Optical spectra displayed in an optical spectrum analyzer with $20 \mathrm{pm}$ resolution. The red dashed line shows the single mode operation of one slave laser; the red continuous line shows the OFC generated when this laser is directly modulated at $500 \mathrm{MHz}$ (gain-switching regime). The blue line depicts the final aggregate of the two OFCs $(500$ and $500.1 \mathrm{MHz}$ repetition frequencies) and the master laser peak (see text for details).

the number of components implicated. The architecture is also fully validated as a spectrometer in order to obtain the absorption and dispersion profile of the molecular transition of $\mathrm{H}^{13} \mathrm{CN}$ at $1538.523 \mathrm{~nm}$.

The basic experimental setup is presented in Fig. 1(a), where two slave lasers are gain-switched to prompt the pulsed regime and, subsequently, a pair of OFCs with different repetition frequencies. Then, two optical pulse trains arise, which are then combined and eventually heterodyned on a photodetector, leading to a RF comb that can be finally digitized. To endow the system with mutual coherence, the output power of a CW master laser is bisected with a 50/50 optical coupler in order to inject both slave lasers, after traversing an optical isolator which suppresses any unsought feedback reflection.

Three discrete mode laser diodes take part in the setup (EP 1538-5-DM-H19-FM, Eblana Photonics Ltd.), with different linewidths $(34 \mathrm{kHz}$ for master, $54 \mathrm{kHz}$ for slave 1 , and $367 \mathrm{kHz}$ for slave 2). A pair of GS-OFCs are generated in the slave lasers with repetition frequencies at $500 \mathrm{MHz}$ and $500.1 \mathrm{MHz}$, respectively. Both slaves laser are biased with the same GS ratios, fixed at $I_{\mathrm{RF}} / I_{\text {bias }} \approx 2.2$ and $I_{\text {bias }} / I_{\mathrm{TH}} \approx 4$ (where $I_{\mathrm{RF}}$ is the RF $\mathrm{CW}$ signal which modulates the laser bias current, $I_{\text {bias }}$, and $I_{\mathrm{TH}}$ refers to the threshold current). The different optical spectra obtained are illustrated in Fig. 1(b). The red dashed line represents the single mode of only one slave laser (for the sake of clarity, the slave counterpart and master laser are both switched off). When this slave is directly modulated at $500 \mathrm{MHz}$, an asymmetric OFC (red continuous line) arises where its peak is located at a higher wavelength (due to GS dynamics). The blue line symbolizes the final combination in the spectra of this OFC with the second GS-OFC from the second slave laser (with repetition frequency of $500.1 \mathrm{MHz}$ ) and the master laser peak before reaching the photodetector. This way, taking advantage of the tuning range of the Discrete Mode lasers (around $4 \mathrm{~nm}$ ), the wavelength emission of the three lasers is tuned to create a combined optical spectrum where the master is located at a lower wavelength and the OFCs with slightly different repetition rates at higher wavelengths.

When the two optical signals are recombined, they interfere and are detected by an InGaAs photodetector (PDA10CF, Thorlabs Inc.). The output signal is thus a pulse train (interferogram) where the time spacing between bursts corresponds to the inverse of the difference in the OFCs repetition frequency $\left(1 / \Delta f_{\text {REP }}, 10 \mu\right.$ s in this case), as can be seen in Fig. 2(a). The eventual Fourier transform of the time-domain signal provides the frequency-domain picture, where a RF comb with line spacing $\Delta f_{\text {REP }}$ sprouts. With this arrangement, the RF comb encompasses around 100 lines with SNR over $100(20 \mathrm{~dB})$ which correspond to the part of the optical spectrum situated at higher wavelengths [Fig. 2(b)].

At this point, it is important to remark on the absence of any frequency shift stage to avoid beat-note averaging between upper and lower sidebands, as in electro-optic dual-comb schemes implemented with one CW laser. In these architectures, this process was usually carried out by an acousto-optic modulator $(\mathrm{AOM})$ to induce a frequency shift in one of the OFCs and unequivocally map every spectral feature into the RF domain. In the case of GS-OIL dual-comb schemes,

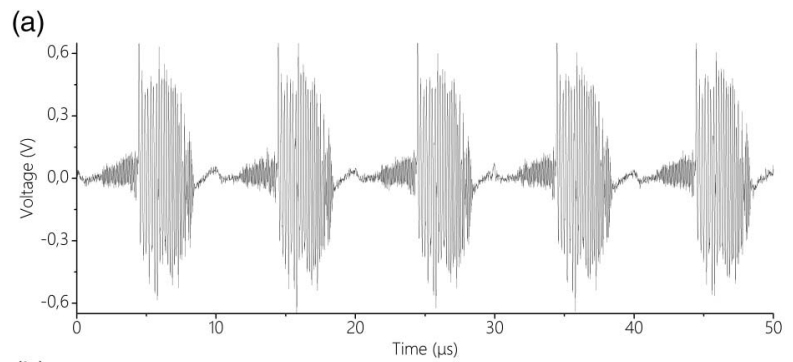

(b)

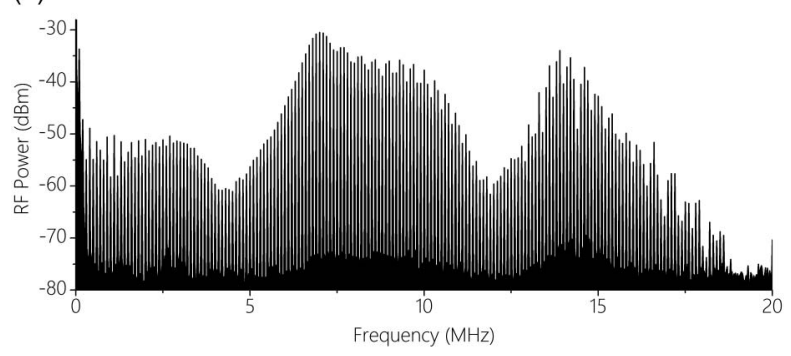

Fig. 2. GS-OIL based dual-comb. Temporal and RF domain. (a) Time-domain interferogram arising after the two pulse trains in the optical domain impinge on a photodetector, with a separation between adjacent pulses of $10 \mu \mathrm{s}$. (b) RF comb measured using an electrical spectrum analyzer. It is possible to identify more than 100 lines with SNR over $100(20 \mathrm{~dB})$ in agreement with the region of the optical spectrum which has been conveniently placed at higher wavelengths. 
this issue can be circumvented by generating highly asymmetric combs (in which the vast majority of comb lines appear at wavelengths above the wavelength emission of the master) and with appropriate optical injection of the master laser. This way, the largest part of the optical spectrum can be mapped from the optical to the RF domain and the overlapping of lower and upper sidebands can be reduced to a minimum, only by precisely tuning the wavelength of the master laser. The existence of a comb-tooth resolved spectra in this domain with sub-Hertz residual linewidths is clear evidence of the effective lock of both slaves to the master.

Nonetheless, this process of overlapping reduction by selecting the master injection wavelength conveys some limitations which must be borne in mind for dual-comb schemes. The fundamental role of the master in this architecture stems from providing mutual coherence between a pair of OFCs which otherwise would not lead to comb-tooth resolved spectra and high resolution. For that purpose, the influence of the master laser wavelength emission has been analyzed in terms of number of lines in the RF comb and their SNR while maintaining the wavelength emission of the slaves at a fixed value. To that end, an offset to a selected wavelength emission of the master [about $1538.2 \mathrm{~nm}$, see Fig. 1(b)] has been induced by slightly adjusting the temperature with a standard laser diode controller. The results of this experiment are illustrated in Fig. 3, where the SNR of the RF comb teeth is depicted when introducing a continuous offset value with respect to the reference (master wavelength offset $=0.00 \mathrm{~nm}$ ) over a RF bandwidth of $20 \mathrm{MHz}$. Additionally, in the interest of clarity, seven RF combs are also 2D-plotted for a set of discrete wavelength offset values.

This being said, one can see that there exists an optimal injection point in which the number of lines (and SNR) of the RF comb is maximum. On the other hand, several non-ideal cases can be identified. By detaching the master from the slave OFCs (negative offset in Fig. 3) the SNR of the teeth deteriorates, whereas by placing it closer to the OFCs (positive offset) the effect of the edge-line injection vanishes, leading to a narrower RF comb with lower SNR. It is also noteworthy to mention that the level of injection ratio did not have any effect on the depicted results as long as the master laser had enough power to lock both slave OFCs. The authors also want to point out the importance of the diode laser technology, which may bring about a different outcome in the process of optical injection on gain-switched lasers and, eventually, in the RF comb generated.

To validate the dual-comb architecture, the diagram presented in Fig. 1(a) is conveniently modified to be used as a spectrometer, where the device under test (DUT) is inserted into one of the branches of the interferometer (Fig. 4). Moreover, supplementary fiber polarization controllers placed between all the lasers help to ensure proper coupling of the injected light into the slave lasers and also suitable matching of the OFCs polarization state for interference. In this case, the DUT encompasses a $55 \mathrm{~mm}$ length gas cell filled with pure $\mathrm{H}^{13} \mathrm{CN}$ at 100 Torr (HCN-13-H(5.5)-100-FCAPC, Wavelength References Inc.) together with an optical switch (OSW-2X2BA, Fiberstore Inc.) which can permit one of the OFCs to bypass the cell. The same optimum OFC and eventual RF comb as described above and illustrated in Fig. 3 were employed for the validation. After low-pass filtering with standard RF filters, the digitization of the time-domain



Fig. 3. Impact of the wavelength emission of the master laser on the recovered RF comb. The background color map shows the evolution of the SNR of the RF comb lines over a $20 \mathrm{MHz}$ bandwidth when the wavelength of the master varies with respect to an initial point (chosen arbitrarily as zero-point). In the foreground, the 2D-plots of seven RF combs for several cases of wavelength offset are shown. Either a negative offset (master farther from the slaves OFCs) or positive offset (master closer to the slave OFCs) lead to lower SNR in comparison to the zero-point case [Fig. 1(b)].

interferogram was carried out by a 14-bit waveform digitized board (PDA14, Signatec Inc.) sampling at a rate of $64 \mathrm{MS} / \mathrm{s}$. All RF signals were generated by the same phase coherent synthesizer. The processing method relies on a multichannel digital lock-in detection system further detailed elsewhere [22], which allows for the retrieval of the information associated with every spectral point, namely amplitude and phase.

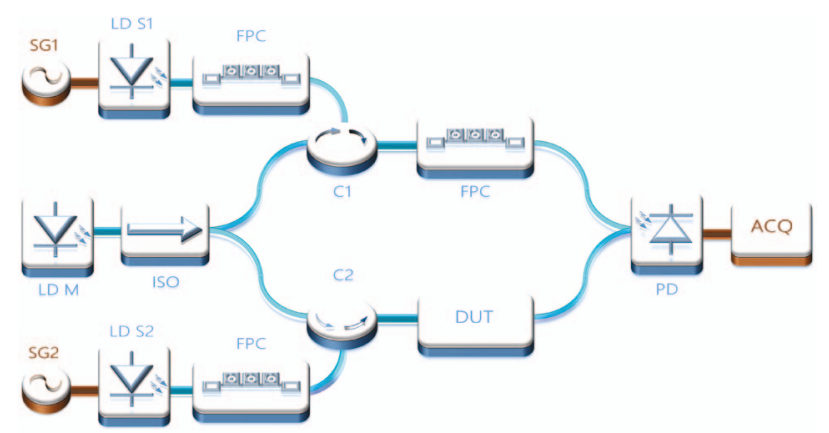

Fig. 4. Adapted GS-OIL dual-comb scheme for dispersive-based spectroscopy measurements. The device under test is incorporated in one of the arms of the interferometer to extract both relative amplitude and phase of the OFC lines which pass through the DUT. The addition of fiber polarization controllers (FPC) also helps in the injection process and in the matching of both OFCs. 


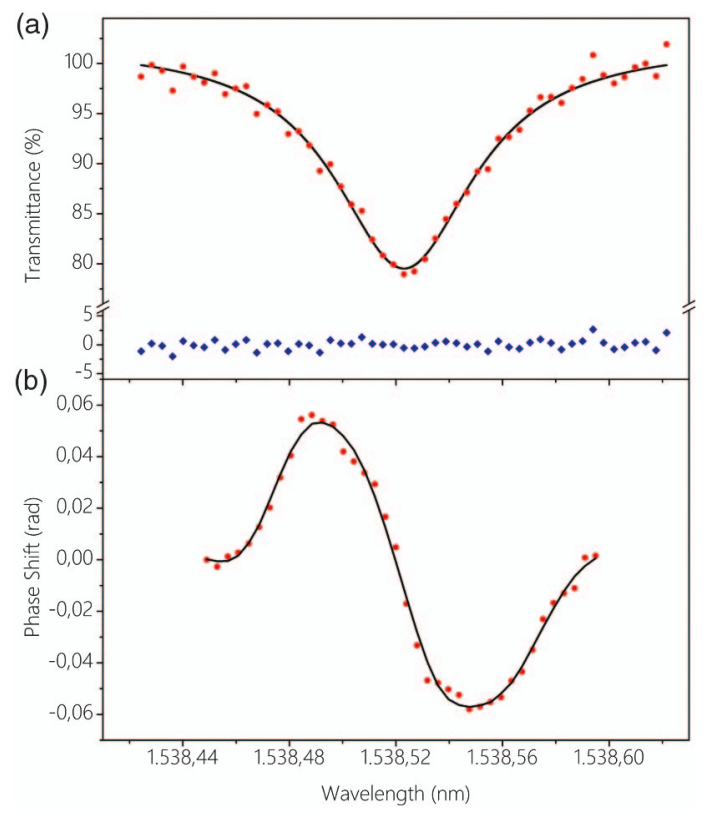

Fig. 5. Absorption (a) and dispersion (b) profile of the measured rovibrational transition of the $2 \nu 3$ band of $\mathrm{H}^{13} \mathrm{CN}$ at $1538.523 \mathrm{~nm}(\mathrm{R}(5)$ line). Average of 10 spectra over an integration time of $10 \mathrm{~ms}$. (a) The continuous line shows the Voigt fit of the ratio between the amplitudes of the comb teeth when the OFC traverse the gas cell (dots) along with the residuals between measured spectra and fit (blue diamonds), which show a standard deviation of $0.84 \%$. (b) Experimental profile (dots), along with box-smoothing (continuous line).

The outcome of the processing method unveils the absorption and dispersion profile of the target spectral feature, as depicted in Fig. 5. In this example, ten spectra were averaged over an integration time of $10 \mathrm{~ms}$. The absorption profile was successfully fit by a Voigt profile [Fig. 5(a)], whereas the dispersive profile was box-smoothed to filter out multiplicative phase noise [29] [Fig. 5(b)]. The values of transmittance in the center of the line $(\sim 78 \%)$, as well as the linewidth in both cases $(\sim 8 \mathrm{GHz})$, show agreement with the standard value reported [30]. For this scheme, the value of the noise-equivalent absorption (NEA, $\left.\left(L_{\mathrm{abs}} \mathrm{SNR}\right)^{-1}(T / M)^{1 / 2}\right)$ over the $1 \mathrm{~s}$ averaging time is $5.75 \times 10^{-6} \mathrm{~cm}^{-1} \mathrm{~Hz}^{-1 / 2}$, where $L_{\text {abs }}=5.5 \mathrm{~cm}$, $\mathrm{SNR}=100, T=1 \mathrm{~ms}$, and $M=100$.

In summary, the present work has explored the capabilities of GS and OIL to create optimized dual-comb architectures in terms of number of lines and SNR. As we have demonstrated, not only does this architecture preserve all the classic advantages of electro-optic dual-comb architectures, but it also allows for a reduction in the number of components involved, only by efficiently applying the techniques on which this system is based. The proficiency of the system has also been further explored according to the dispersive arrangement, which has permitted the recovery of the phase profile of the molecular transition of $\mathrm{H}^{13} \mathrm{CN}$ at $1538.523 \mathrm{~nm}$. These results prove that the GS-OIL dual-comb architecture possesses promising implementation capabilities from mid-infrared to near ultraviolet due to the absence of wavelength-restrictive optical elements, as well as the possibility to be incorporated in photonic integrated circuits (PICs) using generic platforms or taking part in compact, cost-efficient setups for field applications.
Funding. Ministerio de Economía y Competitividad (MINECO) (RTC-2014-2661-7, TEC-2014-52147-R); Spanish Ministry of Education, Culture, and Sports (FPU014/06338).

\section{REFERENCES}

1. K. Y. Lau, Appl. Phys. Lett. 52, 257 (1988).

2. P. P. Vasil'ev, I. H. White, and J. Gowar, Rep. Prog. Phys. 63, 1997 (2000).

3. S. Kobayashi and T. Kimura, IEEE J. Quantum Electron. 16, 915 (1980).

4. A. Takada and W. Imajuku, IEEE Photon. Technol. Lett. 9, 1328 (1997).

5. R. T. Ramos, P. Gallion, D. Erasme, A. J. Seeds, and A. Bordonalli, Opt. Lett. 19, 4 (1994).

6. A. M. Clarke, P. M. Anandarajah, and L. P. Barry, IEEE Photon. Technol. Lett. 16, 2344 (2004).

7. R. Zhou, S. Latkowski, J. O'Carroll, R. Phelan, L. P. Barry, and P. Anandarajah, Opt. Express 19, B415 (2011).

8. P. M. Anandarajah, R. Maher, Y. Q. Xu, S. Latkowski, J. O'Carroll, S. G. Murdoch, R. Phelan, J. O'Gorman, and L. P. Barry, IEEE Photon. J. 3, 112 (2011)

9. R. Zhou, T. N. Huynh, V. Vujicic, P. M. Anandarajah, and L. P. Barry, Opt. Express 22, 8120 (2014).

10. S. P. ÓDúill, P. M. Anandarajah, R. Zhou, and L. P. Barry, Appl. Phys. Lett. 106, 211105 (2015).

11. M. D. Gutierrez Pascual, R. Zhou, F. Smyth, P. M. Anandarajah, and L. P. Barry, Opt. Express 23, 23225 (2015).

12. P. M. Anandarajah, K. Shi, J. O'Carroll, A. Kaszubowska, R. Phelan, L. P. Barry, A. D. Ellis, P. Perry, D. Reid, B. Kelly, and J. O'Gorman, Opt. Express 17, 12668 (2009).

13. E. Prior, C. de Dios, Á. R. Criado, M. Ortsiefer, P. Meissner, and P. Acedo, IEEE Photon. Technol. Lett. 26, 2118 (2014).

14. R. Zhou, P. M. Anandarajah, D. Gutierrez Pascual, J. O'Carroll, R. Phelan, B. Kelly, and L. P. Barry, in Optical Fiber Communication Conference, OSA Technical Digest (Online) (Optical Society of America, 2014), paper Th3A.3.

15. J. K. Alexander, P. E. Morrissey, H. Yang, M. Yang, P. J. Marraccini, B. Corbett, and F. H. Peters, Opt. Express 24, 7960 (2016).

16. M. D. Gutierrez, J. Braddell, F. Smyth, and L. P. Barry, in 18th European Conference on Integrated Optics (2016), paper o-01.

17. B. Jerez, P. Martín-Mateos, E. Prior, C. de Dios, and P. Acedo, Opt. Express 24, 14986 (2016).

18. S. Schiller, Opt. Lett. 27, 766 (2002).

19. F. Keilmann, C. Gohle, and R. Holzwarth, Opt. Lett. 29, 1542 (2004).

20. I. Coddington, W. C. Swann, and N. R. Newbury, Phys. Rev. A 82, 043817 (2010).

21. D. A. Long, A. J. Fleisher, K. O. Douglass, S. E. Maxwell, K. Bielska, J. T. Hodges, and D. F. Plusquellic, Opt. Lett. 39, 2688 (2014).

22. P. Martin-Mateos, M. Ruiz-Llata, J. Posada-Roman, and P. Acedo, IEEE Photon. Technol. Lett. 27, 1309 (2015).

23. P. Martín-Mateos, B. Jerez, and P. Acedo, Opt. Express 23, 21149 (2015).

24. G. Millot, S. Pitois, M. Yan, T. Hovhannisyan, A. Bendahmane, T. W. Hänsch, and N. Picqué, Nat. Photonics 10, 27 (2016).

25. A. J. Fleisher, D. A. Long, Z. D. Reed, J. T. Hodges, and D. F. Plusquellic, Opt. Express 24, 10424 (2016).

26. P. Martín-Mateos, B. Jerez, E. Prior, C. de Dios, and P. Acedo, in Conference on Lasers and Electro-Optics, OSA Technical Digest (Online) (Optical Society of America, 2016), paper AM2J.5.

27. N. Kuse, A. Ozawa, and Y. Kobayashi, Opt. Express 21, 11141 (2013).

28. V. Durán, S. Tainta, and V. Torres-Company, Opt. Express 23, 30557 (2015).

29. N. R. Newbury, I. Coddington, and W. Swann, Opt. Express 18, 7929 (2010).

30. W. C. Swann and S. L. Gilbert, J. Opt. Soc. Am. B 22, 1749 (2005). 